\title{
SUB-POISSONIAN LIGHT AND PHOTOCURRENT SHOT-NOISE SUPPRESSION IN CLOSED OPTO-ELECTRONIC LOOP
}

\author{
A.V.Masalov, A.A.Putilin, M.V.Vasilyev \\ Lebedev Physical Institute, Leninsky pr.53, 117924 Moscow, Russia
}

\begin{abstract}
We examine experimentally photocurrent noise reduction in the opto-electronic closed loop. Photocurrent noise density $12.5 \mathrm{~dB}$ below the shot-noise level was observed. So large suppression was not reached in previous experiments $[1,2,3,4]$ and can not be explained in terms of an ordinary sub-Poissonian light in the loop. We propose the concept of anticorrelation state for the description of light in the loop.
\end{abstract}

We study closed opto-electronic loop consisting of light source (single-mode, single-frequency argon laser with $1 \mathrm{~W}$ output power at the wavelength $514 \mathrm{~nm}$ ), clectro-optical modulator with halfwavelength voltage $170 \mathrm{~V}, p-i-n$ photodiode having quantum efficiency 0.68 and electronic feedback circuit including two amplifiers with total $62-63 \mathrm{~dB}$ amplification in the $1-100 \mathrm{MHz}$ frequency range and noise temperature less than $300 \mathrm{~K}$ (Fig.1). The modulator opcrates in the regime of the amplitude modulation, where feedback strength $A$ is proportional to the light power and can be easily changed. The feedback strength has been controlled by the value of average photocurrent: $A /\langle i\rangle=0.6-0.7 \mathrm{~m} \Lambda$.

Experimental noise spectra of the feedback photodiode current (Fig.2) are in a good agreement with formula

$$
\frac{\left\langle\delta i^{2}\right\rangle}{\left\langle\delta i_{S N L}^{2}\right\rangle}=\frac{1}{\left|1+\lambda c^{2 \pi j f r}\right|^{2}}
$$

where $\left\langle\delta i_{S N L}^{2}\right\rangle$ is the standard shot-noise level, $\tau$ is loop round-trip time. Eliminating the noise amplification areas by a narrow-bandpass filter wc have obtained the photocurrent noise reduction up to $12.5 \mathrm{~dB}$ (=factor 17.8), i.e. $94.4 \%$ of standard shot noise has been suppressed (Fig.3). This noise reduction is beyond the limit imposed by non-unity quantum efficiency of the feedback photodiode: $\left\langle\delta i^{2}\right\rangle /\left\langle\delta i_{S N L}^{2}\right\rangle$ should be larger than $1-\eta$. Photocurrent noise reduction does not depend on the quantum efficiency of the feedlsack photodiode and can be made arbitrary large (Fig.4). Our data can not be explained in ternis of an ordinary sub-Poissonian light in the loop and require an introduction of the new concept of lightit.

The light beam extracted from the loop cxhibits a noise above the standard quantum limit (Fig.5). This noise is shown in Fig.6 versus the fecdback strength for two values of the feedback photodiode quantum efficiency. The data are in a good agrecment with the theory [5], shown by solid line.

To describe the state of light in the loop we offer the concept of anticorrclation state: the temporal variations of average light intensity produced by electrically driven modulator are anticorrelated to its quantum fluctuations. Due to this anticorrelation the total fluctuations of in-loop 
light intensity can be suppressed:

$$
\frac{\left\langle\delta I^{2}\right\rangle}{\left\langle\delta I_{S Q L}^{2}\right\rangle}=\frac{1+|A|^{2} \frac{(1-\eta)}{\eta}}{|1+A|^{2}}
$$

where $\left\langle\delta I_{S Q L}^{2}\right\rangle$ is the standard quantum level. In addition the part of the intensity modulation component is a posteriori anticorrelated to photocurrent fluctuations arising in the diode due to non-unity quantum efficiency. Thus the photocurrent noise is suppressed, and this suppression is more effective than in the light because the closed opto-electronic loop is a device stabilizing the fluctuations of the current at the modulator. The light here serves only as a feedback signal carrier.

When a beam splitter is inserted into the loop to extract the light, the intensity modulation component contains a term anticorrelated to the quantum fluctuations brought by the beam splitter. However in the extracted beam the corresponding quantum fluctuations are correlated rather than anticorrelated to this modulation term. 'Tlicrefore extracted bean noise is always super-Poissonian:

$$
\frac{\left\langle\delta I^{\prime 2}\right\rangle}{\left\langle\delta I_{S Q L}^{\prime 2}\right\rangle}=1+\frac{A}{1+A} \frac{1-T}{T^{\prime} \eta}
$$

The principal difference between the anticorrelation light and ordinary sub-Poissonian light is seen from the following example: the ordinary sub-Poissonian light decreases its sub-Poissonian quality when passes through a beam splitter; the anticorrelation light with sub-Poissonian noise passes through a beam splitter and improves its sub-Poissonian factor while the reflected beam gains super-Poissonian statistics. In principle, this paradoxical situation may be observed by QND measurements.

\section{References}

[1] S.Mashida, Y.Yamamoto. Optics Commun. 57, 290 (1986).

[2] Y.Yamamoto, N.Imoio, S.Mashida. Pliys. Rev. A 33, 3213 (1986).

[3] P.R.Tapster, J.G.Rarity, J.S.Satchell. Phys. Rev. A 37, 2963 (1988).

[4] Ya.A.Fofanov. Kvantovaya Elektronika (Russian) 16, 2593 (19S9).

[5] J.II.Shapiro, G.Saplakoglu, S.-'T.Ilo, P.Kunar, B.E.A.Saleh, M.C.Teicl. JOSA B 4, 1604 (1987). 

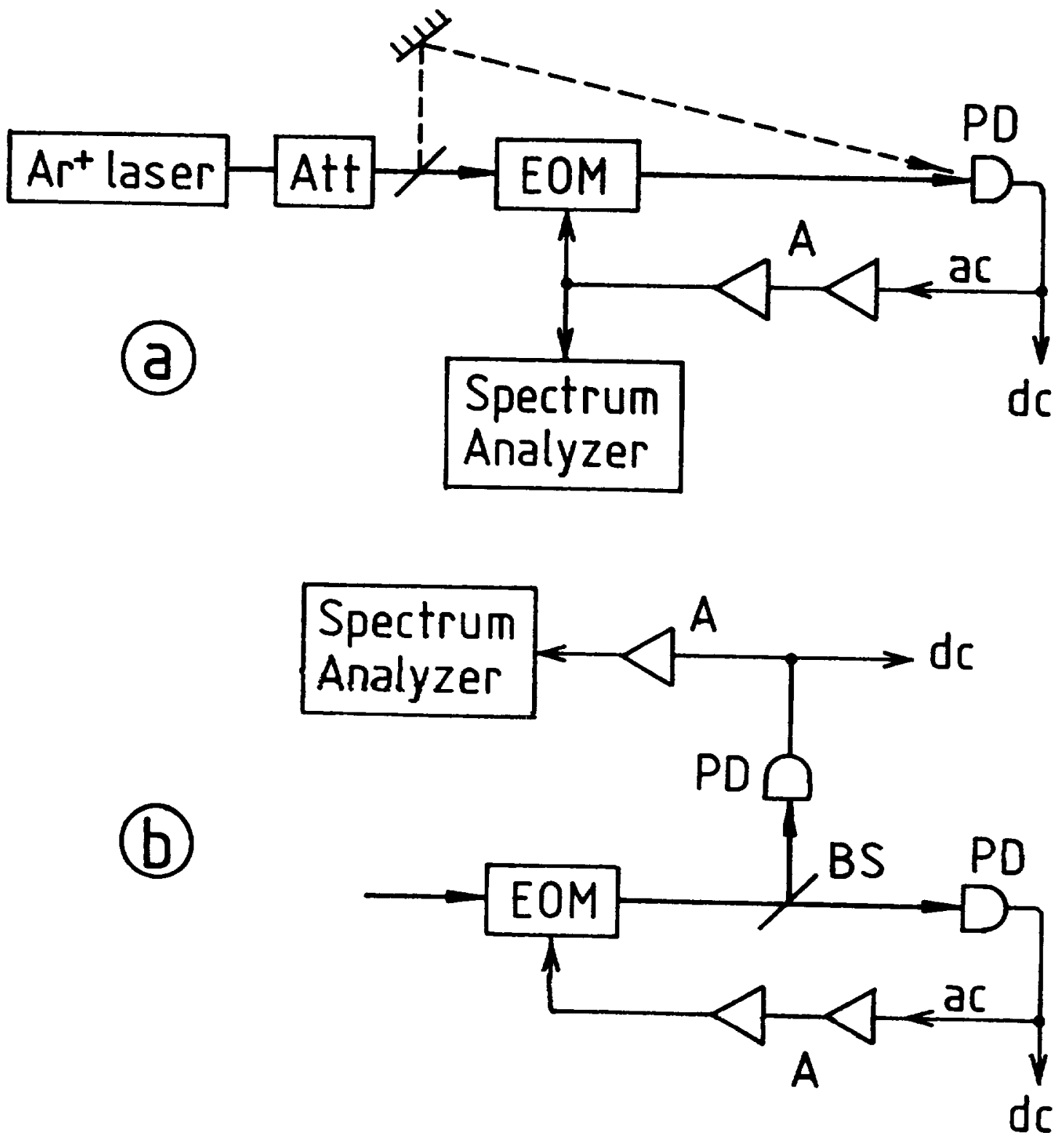

Fig. 1. Experimental setup: $a$ - meisurements of photocurrent noise in the closed opto-electronic loop, $b$ - the same for the beam extracted from the loop. PD - photodiodes, EOM - electro-optical modulator, BS - bcam splitter, $A$ - amplificrs, $A t t$ attenuator. 


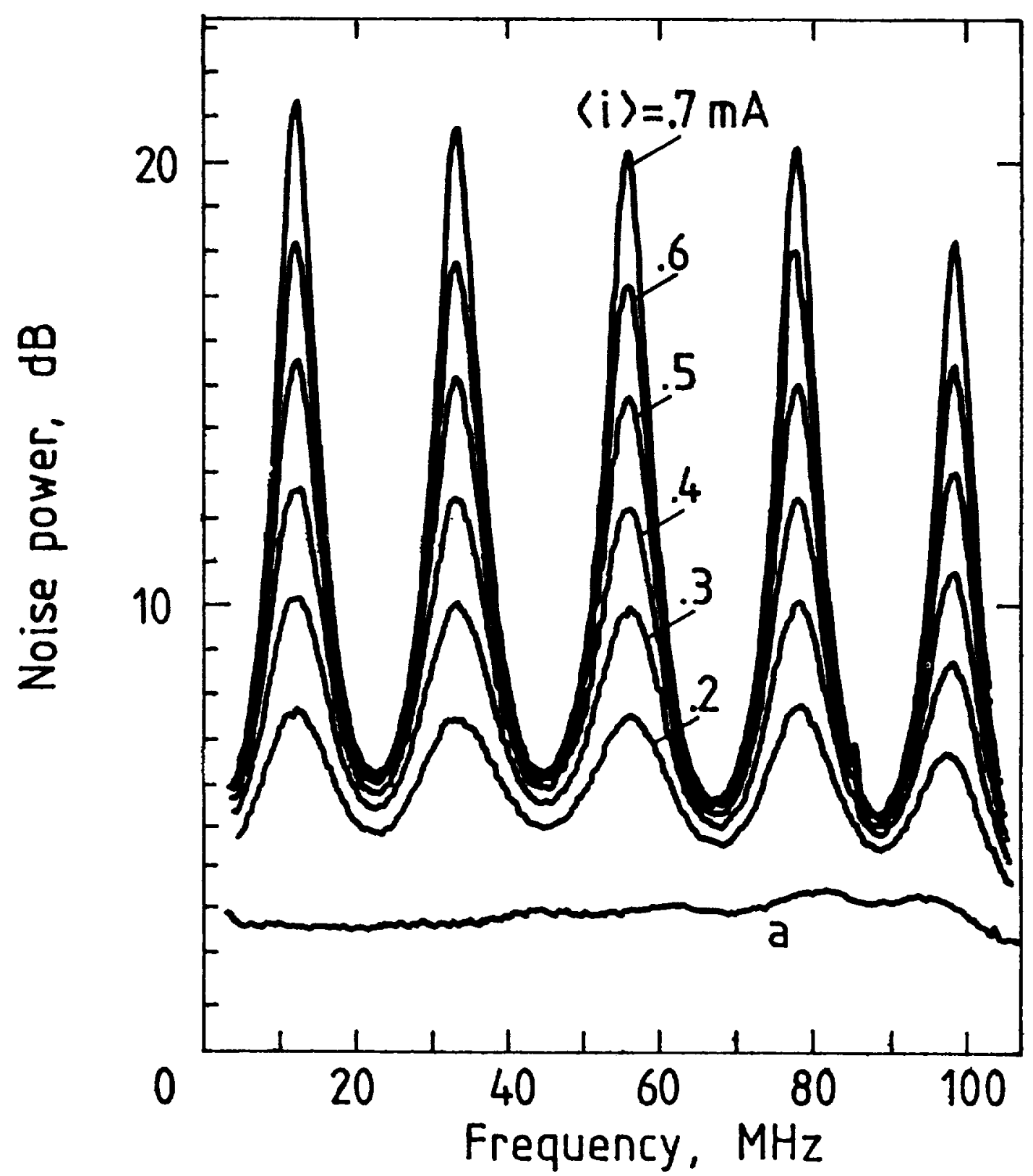

Fig. 2. Noise spectra of the fecdback photodiode current at different values of the feedback strength; $a$ - amplifier noise, loop round-trip time $\tau=47 n s$. 


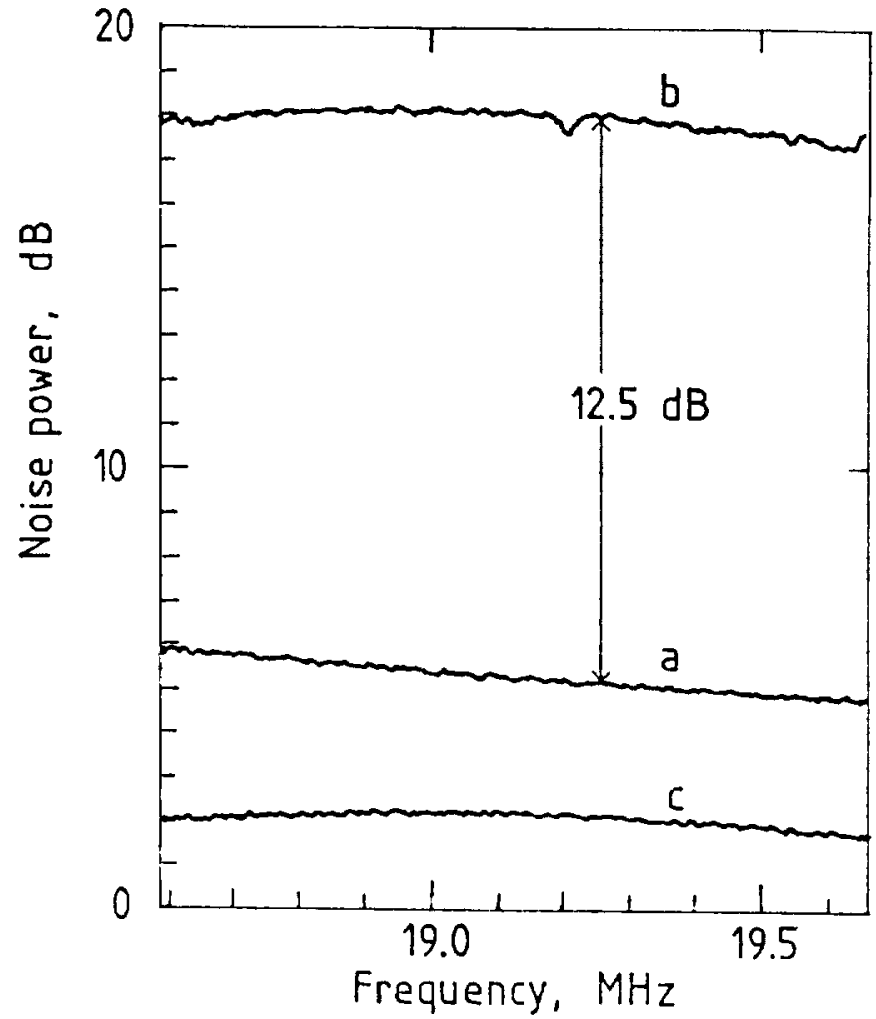

Fig. 3. $a$ - photocurrent noise spectral density in the loop with a narrow-band filter in the feedback circuit, $b$ - standard shot-noise level, $c$ - amplifier noise.

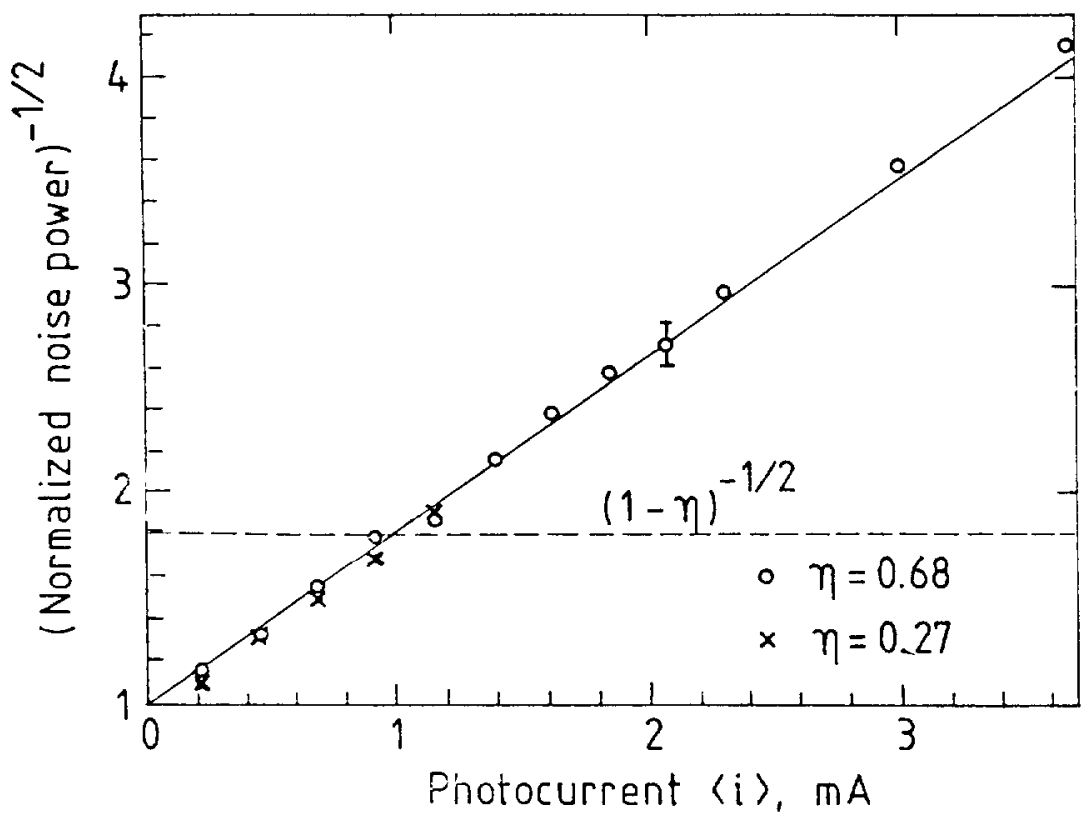

Fig. 4. Shot-noise suppression factor at the frequency $19.2 \mathrm{MIIz}$ versus feedback strength in the units of average photocurrent. Dashed line is the limit due to the quantum efficiency of the feedback photodiode $\eta=0.68$. 


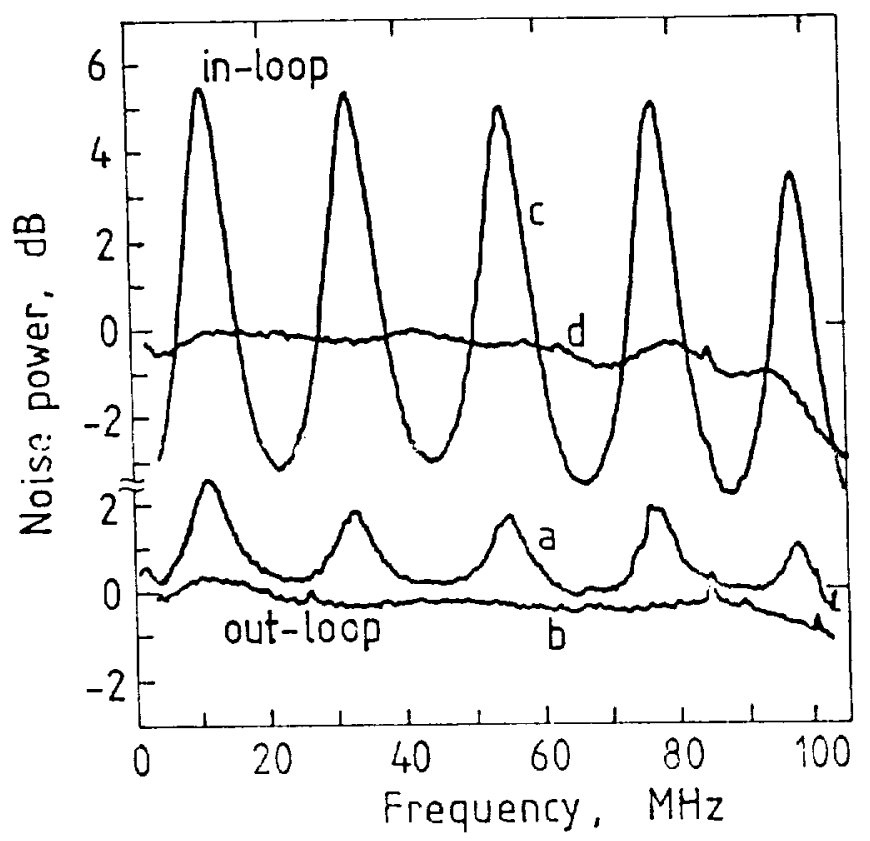

Fig. 5. Photocurrent noise spectrum for the photodiode in the extracted beam $(a)$ and the relevant standard shot-noise level $(b) ; c$ and $d$ are those for feedback photodiode.

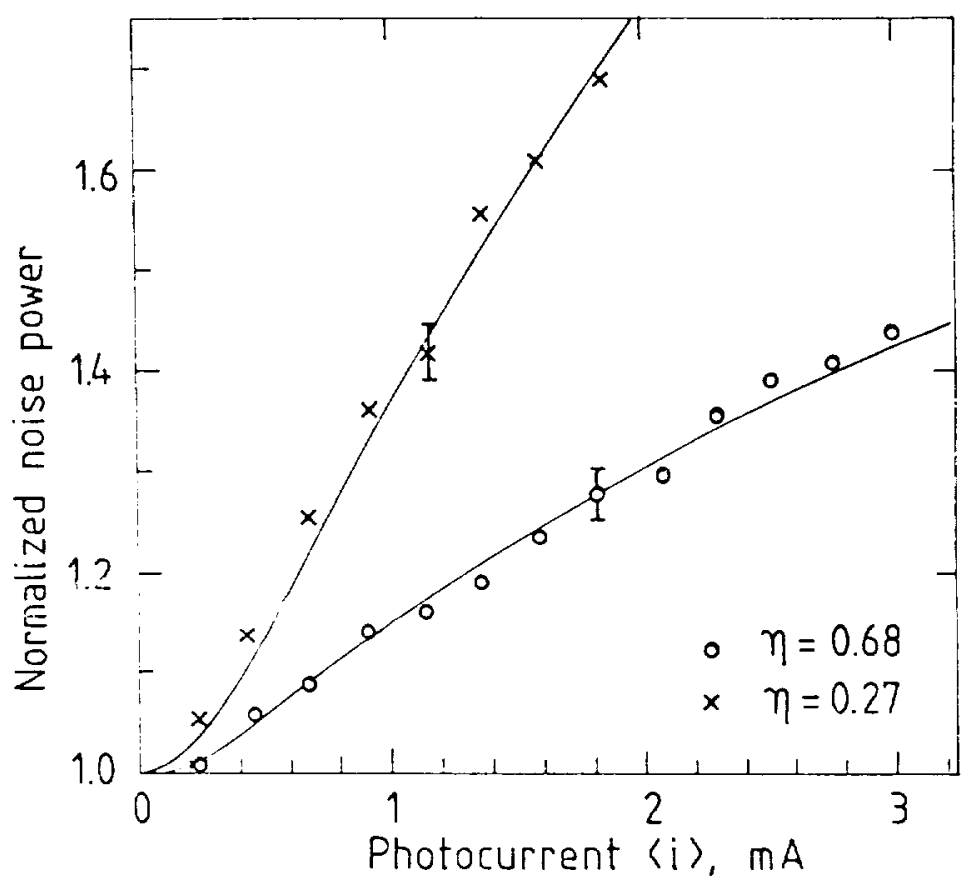

Fig. 6. Noise spectral density of the extracted beam photocurrent, normalized to the standard shot-noise level, versus the feedback strength (in the units of the average fcedback photocurrent). 